\title{
Vav1 inhibits RANKL-induced osteoclast differentiation and bone resorption
}

\author{
Jin Sun Jang ${ }^{1, \#}$, In Soon Kang ${ }^{1, \#}$, Young-Nam Cha ${ }^{1}$, Zang Hee Lee ${ }^{2}$, Mary C Dinauer ${ }^{3}$, Young-June Kim ${ }^{4}$ E Chaekyun Kim $^{1, *}$ \\ ${ }^{1}$ Laboratory for Leukocyte Signaling Research, Department of Pharmacology, Inha University School of Medicine, Incheon 22212, Korea, \\ ${ }^{2}$ Department of Cell and Developmental Biology, Dental Research Institute, School of Dentistry, Seoul National University, Seoul 03080, \\ Korea, ${ }^{3}$ Department of Pediatrics, Washington University School of Medicine, St Louis, MO 63100, USA, ${ }^{4}$ Department of Microbiology and \\ Immunology, Indiana University School of Medicine, Indianapolis, IN 46202, USA
}

Vav1 is a Rho/Rac guanine nucleotide exchange factor primarily expressed in hematopoietic cells. In this study, we investigated the potential role of Vav1 in osteoclast (OC) differentiation by comparing the ability of bone marrow mononuclear cells (BMMCs) obtained from Vav1-deficient $\left(\mathrm{Vav1}^{-1-}\right)$ and wild-type (WT) mice to differentiate into mature OCs upon stimulation with macrophage colony stimulating factor and receptor activator of nuclear kappa $B$ ligand in vitro. Our results suggested that Vav1 deficiency promoted the differentiation of BMMCs into OCs, as indicated by the increased expression of tartrate-resistant acid phosphatase, cathepsin $\mathrm{K}$, and calcitonin receptor. Therefore, Vav1 may play a negative role in $\mathrm{OC}$ differentiation. This hypothesis was supported by the observation of more OCs in the femurs of Vav1 ${ }^{-1-}$ mice than in WT mice. Furthermore, the bone status of $\mathrm{Vav1} 1^{-1-}$ mice was analyzed in situ and the femurs of $\mathrm{Vav1}^{-1-}$ mice appeared abnormal, with poor bone density and fewer number of trabeculae. In addition, Vav1-deficient OCs showed stronger adhesion to vitronectin, an $\alpha_{v} \beta_{3}$ integrin ligand important in bone resorption. Thus, Vav1 may inhibit OC differentiation and protect against bone resorption. [BMB Reports 2019; 52(11): 659-664]

\section{INTRODUCTION}

Vav proteins are Dbl family guanine nucleotide exchange factors (GEFs) that activate Rho family GTPases by stimulating the release of GDP to allow GTP binding. There are three

${ }^{*}$ Corresponding author. Tel: +82-32-860-9874; Fax: +82-32-8858302; E-mail: chaekyun@inha.ac.kr

${ }^{\#}$ These authors contributed equally to this work.

https://doi.org/10.5483/BMBRep.2019.52.11.013

Received 7 January 2019, Revised 8 February 2019, Accepted 24 April 2019

Keywords: $\alpha_{v} \beta_{3}$ integrin, Guanine nucleotide exchange factor, Osteoclast, RANKL, Vav1 members of the Vav protein family, Vav1, Vav2, and Vav3. Vav1 is expressed exclusively in hematopoietic cells (1), whereas Vav2 and Vav3 are expressed ubiquitously. Vav proteins are involved in essential cellular processes such as differentiation, proliferation, cytoskeletal organization, and vesicle trafficking. In addition, these proteins play an essential role in the coupling of $\beta_{2}$ integrin to Rho GTPases and regulate multiple integrin-induced events in leukocyte adhesion, migration, and phagocytosis $(2,3)$. Vav proteins are activated by growth factor receptors, chemokine receptors and integrins, and regulate multiple cellular processes such as osteoclast (OC) differentiation via the activation of Rho GTPases (4). Although Vav proteins share redundant functions, they also exhibit unique, isoform-specific roles. Vav1 regulates actin polymerization, cytoskeletal organization, adhesion, migration, and phagocytosis in hematopoietic cells. Vav1 is also required for optimal spreading and regulation by $\alpha_{11 b} \beta_{3}$ integrin (5), and upregulates $\alpha_{4} \beta_{1}$-mediated T lymphocyte adhesion $(6,7)$ and $\beta 1$ integrin-mediated adhesion in dendritic cells (8).

Bone homeostasis is critical for the maintenance, repair and remodeling of bones, and depends on the balance between bone formation by osteoblasts and bone breakdown by OCs. OCs are a type of bone cell responsible for breaking down bone tissue via a process termed resorption, which occurs through physical association between OCs and the bone matrix $(9,10)$. OCs differentiate from the hematopoietic monocyte/macrophage lineage and are formed by the fusion of mononuclear OC precursor cells into multinucleate cells with abundant cytoplasmic azurophilic granules $(11,12)$. OCs can be produced from bone marrow mononuclear cells (BMMCs) in vitro by several cytokines, such as macrophage colony stimulating factor (M-CSF) and receptor activator of nuclear factor kappa B (NF-kB) ligand (RANKL) (13). OCs must tightly adhere to the bone surface to form a sealing zone which facilitates bone resorption (14). The binding of integrins to their corresponding ligands plays an important role in bone recognition by OCs. The transmembrane integrin $\alpha_{v} \beta_{3}$ is the most abundant integrin in OCs (15), and several studies have reported that $\alpha_{v} \beta_{3}$ integrin mediates the adhesion of OCs to bone surfaces.

ISSN: 1976-670X (electronic edition)

Copyright (c) 2019 by the The Korean Society for Biochemistry and Molecular Biology

(c) This is an open-access article distributed under the terms of the Creative Commons Attribution Non-Commercial License (http://creativecommons.org/licenses/by-nc/4.0) which permits unrestricted non-commercial use, distribution, and reproduction in any medium, provided the original work is properly cited. 
In this study, we explored the possible role of Vav1 in OC differentiation and bone resorption using Vav1-deficient $\left(V_{a v 1}{ }^{-1-}\right)$ mice. BMMCs differentiate into OC precursors in the presence of M-CSF in vitro, and subsequently develop into mature OCs in the presence of M-CSF and RANKL (16). $\mathrm{Vav} 1^{-1-}$ mice were used to evaluate whether $\mathrm{Vav} 1^{-1-}$ BMMCs differed from wild-type (WT) BMMCs in their ability to differentiate into OCs in vitro by measuring various OC-specific markers, such as tartrate-resistant acid phosphatase (TRAP), cathepsin $\mathrm{K}(\mathrm{CTSK})$, calcitonin receptor $(C T R)$, and dendritic cell-specific transmembrane protein (DC-STAMP). We demonstrated that Vav1 suppressed OC differentiation, as indicated by the increased expression levels of OC markers in differentiating $\mathrm{Vav} 1^{-1-}$ cells in vitro. Consistently, TRAP expression was more apparent in the femoral bones of $\mathrm{Vav} 1^{-1-}$ mice than in those of WT mice. We observed abnormalities in the bone mineral density and number of trabeculae in the femurs of $\mathrm{Vav} 1^{-1-}$ mice in vivo. This study reveals the important role of Vav1 in the prevention of bone resorption by suppressing OC differentiation.

\section{RESULTS}

Vav1 suppresses the differentiation of TRAP-positive multinucleated cells

We investigated the role of Vav1 in OC differentiation and bone absorption by comparing $\mathrm{Vav} 1^{-/-}$and WT mice. First,

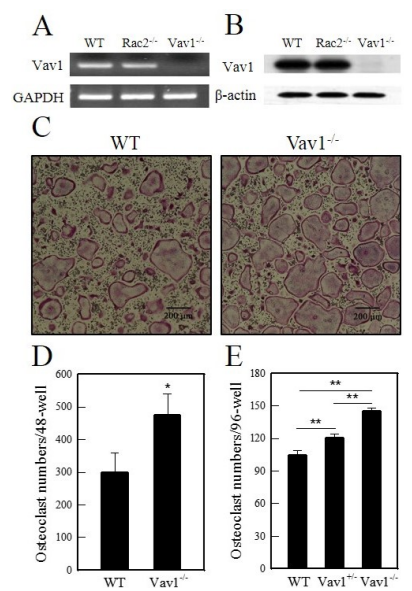

Fig. 1. TRAP-positive OC formation was increased in $\mathrm{Vav} 1^{-1-}$ mice. (A) Total RNA was prepared from the OCs of WT and Vav1 $1^{-1-}$ mice and the absence of Vav1 mRNA was verified by RT-PCR ( $n=6$ ). (B) Vav1 protein deficiency in the OCs was confirmed by western blotting using an anti-Vav1 polyclonal antibody $(\mathrm{n}=3)$. RNA and protein samples from $\mathrm{Rac}^{--}$OCs were used as controls. $(C, D)$ BMMCs were differentiated in the presence of $30 \mathrm{ng} / \mathrm{ml} \mathrm{M-CSF}$ and $50 \mathrm{ng} / \mathrm{ml}$ RANKL, and TRAP-positive multinucleated OCs were counted $(\mathrm{n}=10)$. (E) TRAP-positive OCs differentiated from littermate mice were counted $(\mathrm{n}=3), * \mathrm{P}<0.05$ and $* \mathrm{P}<0.01$. we verified the disruption of Vav1 gene expression in the OCs of the $\mathrm{Vav1}^{-1-}$ mice used in this study (Fig. 1A, B). OC precursors were defined as the cells resulting from a 4-day culture with M-CSF, while mature OCs were defined as the cells obtained from an additional 4 days of culture with M-CSF and RANKL. TRAP activity is an important marker of mature and functional OCs. In the OC precursors, no noticeable differences were observed in the TRAP level between the Vav1 ${ }^{-1-}$ and WT cells (data not shown). However, as the OC precursors differentiated into mature OCs the number of TRAP-positive multinucleated OCs was significantly higher in the $\mathrm{Vav} 1^{-1-}$ cells than in the WT cells (Fig. 1C, D), while heterozygous $\mathrm{Vav} 1^{+/-}$mice produced intermediate levels of TRAP-positive OCs (Fig. 1E). This dose-dependent production of TRAP-positive OCs suggests that Vav1 suppressed OC differentiation. However, the suppressive role of Vav1 seems to be opposite to the effect of Vav3 on OC differentiation reported previously (17). The mechanisms underlying these opposing roles of Vav1 and Vav3 in OC differentiation are not yet understood.

Vav1 suppresses the expression of osteoclastogenic makers We determined the mRNA levels of osteoclastogenic makers in WT and Vav1 ${ }^{-1-}$ OCs. In addition to TRAP, CTSK, CTR, and DC-STAMP are essential for bone resorption $(18,19)$ and their expression levels increase during osteoclastogenesis. Quantitative RT-PCR analysis showed that the mRNA expression of these markers was significantly higher in Vav ${ }^{-1-}$ OCs compared to WT OCs (Fig. 2A-D). Consistent with the increase in the number of TRAP-positive OCs, the enzymatic activity of TRAP was also higher in Vav1 ${ }^{-1-}$ OCs than in WT OCs, confirming the negative effect of Vav1 on OC differentiation (Fig. 2E).

\section{Vav1 suppresses $\alpha_{v} \beta_{3}$-mediated adhesion of OCs}

The binding of integrins to their corresponding ligands plays an important role in the recognition of bone by OCs (3). Integrin $\alpha_{v} \beta_{3}$ is the most abundant integrin in OCs (14). Vav proteins are known to control integrin-mediated cell adhesion through "inside-out" signaling. However, the roles of Vav1 in

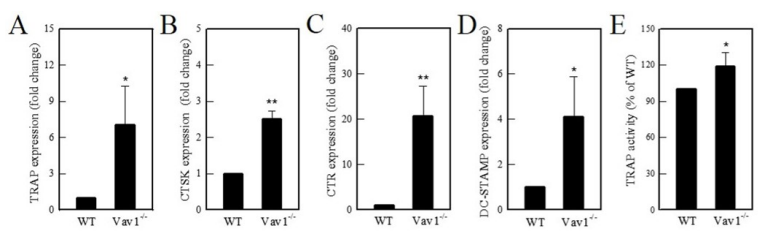

Fig. 2. OC-specific markers and TRAP activity were increased in Vav1 ${ }^{-1-}$ mice. Expression level of mRNA of TRAP (A), CTSK (B), CTR (C), and DC-STAMP (D) in OCs was measured by GRT-PCR $(\mathrm{n}=5)$. (E) TRAP activity in OCs was determined using p-nitrophenyl phosphate as the substrate $(n=3),{ }^{*} \mathrm{P}<0.05$ and $* * P<0.01$. 
integrin $\quad \alpha_{v} \beta_{3}$-mediated cell adhesion remain unclear. Vitronectin is a glycoprotein that binds to integrin $\alpha_{v} \beta_{3}$ as it contains an Arg-Gly-Asp sequence, a specific integrin $\alpha_{v} \beta_{3}$ binding site. Therefore, we examined whether Vav1 was involved in integrin $\alpha_{v} \beta_{3}$-mediated adhesion by comparing the binding ability of $\mathrm{Vav}^{-1-}$ and $\mathrm{WT}$ OC precursors on vitronectin-coated plates. Following the incubation of $\mathrm{OC}$ precursors on vitronectin-coated plates for 10 and $60 \mathrm{~min}$, the number of adhered cells was counted and plotted as a histogram (Fig. 3). The $\mathrm{Vav1}^{-1-}$ OC precursors exhibited greater adherence to the vitronectin-coated plates than the WT cells. We also determined actin ring formation by Alexa-phalloidin staining, with $\mathrm{Vav}^{-1-}$ OCs showing higher levels of actin ring formation than the WT cells (Supplementary Fig. 1). These results indicate that Vav1 suppresses the $\alpha_{v} \beta_{3}$-mediated adhesion of OCs, presumably resulting in the attenuation of bone resorption activity.

\section{Vav1 deficiency reduces bone volume fraction and number of trabeculae in femurs}

As OCs are known to be involved in bone resorption, we compared the bone densities of Vav $1^{-l-}$ and WT mice using $\mu \mathrm{CT}$. The femurs of $\mathrm{Vav}^{-1-}$ mice appeared less dense than those of WT mice (Fig. 4A). To confirm this observation, the ratio of trabecular bone volume to total bone volume (BV/TV, $\%)$, trabecular thickness (Tb.Th, $\mu \mathrm{m})$, trabecular separation (Tb.Sp, $\mu \mathrm{m})$, and the number of trabeculae (Tb.N, $\mathrm{mm}^{-1}$ ) were assessed. Trabeculae are composed of bone cells and collagenous tissue, and are an important skeletal element that supports the framework of organs and body. The BV/TV ratio is an important parameter for evaluating the microstructure of the trabecular bone (20). Vav1 $1^{-1-}$ mice showed significantly lower BV/TV, Tb.Th, and Tb.N than WT mice (Fig. 4B-E), demonstrating that Vav1 prevents OC-mediated reductions in bone mass.

A
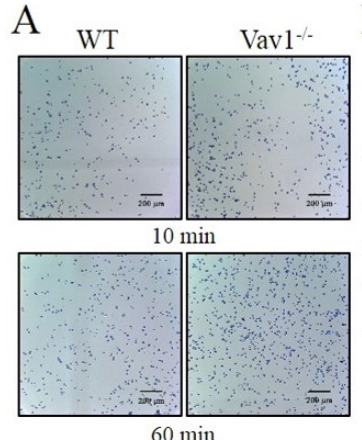

B

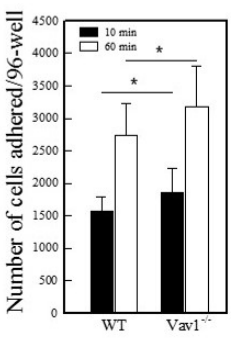

Fig. 3. Adhesion of OC precursors to vitronectin was increased in the absence of Vav1. The ability of OC precursors to adhere to vitronectin was determined in the presence of M-CSF and RANKL for 10 and $60 \mathrm{~min}$ (A), and the number of adhered cells was counted and plotted as a histogram $(B)(n=3), * P<0.05$.

\section{Bones of $\mathrm{Vav1}^{-l-}$ mice contain more TRAP-positive cells than those of WT mice}

As the results obtained with OCs in vitro suggested that Vav1 suppresses OC differentiation, we examined the effects of Vav1 on OC formation in vivo. We compared the number of TRAP-positive cells in the bone sections obtained from Vav1 ${ }^{-1-}$ and WT mice. Consistent with the in vitro results, the femoral bone sections of $\mathrm{Vav} 1^{-1-}$ mice had almost twice as many TRAP-positive cells than those of WT mice (Fig. 4F, G), confirming the suppression of OC differentiation by Vav1 in vivo.

\section{DISCUSSION}

Vav family GEFs are expressed as three isoforms, with Vav3 being the most abundant in OCs (17). Vav3 has been shown to stimulate OC differentiation and bone resorption by enhancing the affinity of integrin $\alpha_{v} \beta_{3}$ for vitronectin (17). Vav3-deficient OCs display defective resorption activity due to impaired signaling downstream of the M-CSF receptor and $\alpha_{v} \beta_{3}$ integrin. As a result, the bones of Vav3-deficient mice exhibit increased density.

In this study, we evaluated the role of Vav1 in OC differentiation. Surprisingly, Vav1 had the opposite effect of Vav3 by suppressing OC differentiation and bone resorption. To clarify the suppressive effect of Vav1 on OC differentiation, we determined the expression and activation levels of kinases known to be involved in OC differentiation, such as mitogen-activated protein kinases (MAPKs) and Akt (21-23). RANK signaling is initiated by cytoplasmic tumor necrosis factor receptor-associated factors that trigger the activation of signaling cascades downstream of these kinases $(24,25)$.
A
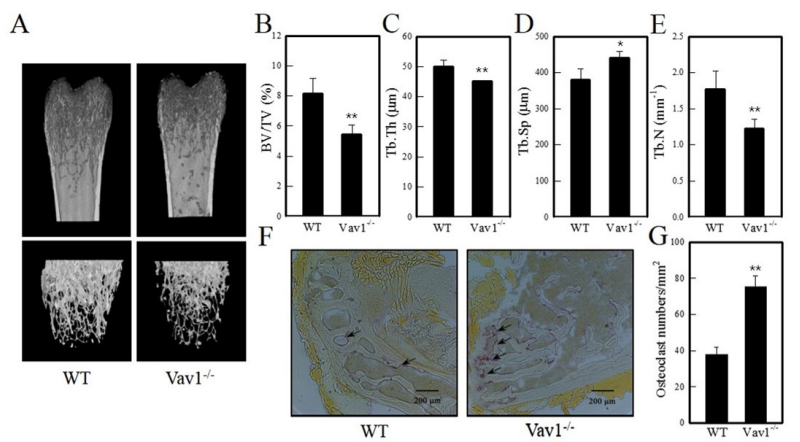

Fig. 4. Vav1 deletion leads to poor bone density in vivo. (A) Microcomputed tomography of the femurs of WT and Vav1 $1^{-/}$ mice (6-wk-old male mice, $\mathrm{n}=4$ per group). (B) Trabecular bone volumes of WT and Vav1 ${ }^{-1-}$ mice were compared using quantitative $\mu \mathrm{CT}$. (C-E) Trabecular bone parameters of WT and Vav1 ${ }^{-1-}$ mice were compared. (F) TRAP-positive cells in the femurs of 24-wk-old male mice were detected ( $n=3$ per group). (G) The number of TRAP-positive cells in selected femur fields was counted $(\mathrm{n}=3), * \mathrm{P}<0.05$ and $* * \mathrm{P}<0.01$. 
MAPKs play important roles in osteoclastogenesis and the expression of MAPKs has been shown to increase in response to RANKL in RAW 264.7 cells (21). Osteoclastogenesis is regulated by the balance between extracellular signalingregulated kinase (ERK) and p38 MAPK signaling. ERK inhibitors enhance OC differentiation, whereas p38 MAPK inhibitors suppress OC differentiation (21). In this study, the activation of MAPKs by M-CSF and RANKL was inconsistent and the activation of MAPKs and Akt was unimpaired in Vav $1^{-1-}$ OCs (data not shown). These results were consistent with our finding that MAPKs signaling was unaltered in the neutrophils of $\mathrm{Vav1}^{-1-}$ mice (data not shown).

The tentative targets for Vav1 are Rac1, Rac2, and RhoG (26), whereas Vav2 and Vav3 have a higher activity towards RhoA, RhoB, and RhoG $(4,27)$. RANKL selectively activates Rac1; hence, Rac1 may be a key regulator of OC differentiation via the activation of NF-KB (28). Vav1 is known to preferentially activate $\operatorname{Rac} 1(26,29)$; thus, we investigated Rac activation in $\mathrm{Vav}^{-/-}$OCs using an affinity precipitation assay for Rac-GTP (22). The levels of active Rac-GTP decreased with OC differentiation; however, no difference in Rac activation was observed between WT and Vav1 $1^{-1-}$ OCs (data not shown). These results were inconsistent with previous studies, which showed that RANKL selectively activates Rac1 but not Rac2, and that Rac1 selectively regulates OC differentiation $(28,30)$. To determine the GEF role of Vav1 on hematopoietic-specific Rac2, we differentiated the BMMCs of Rac2 ${ }^{-1-}$ mice (31) into OCs. The number of OCs in Rac2 ${ }^{-1-}$ cells decreased to $40 \%$ of the number in WT cells, whilst, it increased in Vav1 $1^{-1-}$ cells (data not shown). We expected that Vav1 and Rac2 would exert similar effects on OC differentiation; however, Vav1 suppressed OC differentiation whilst Rac2 was essential. This suggests that Vav1 is not an upstream regulator of Rac2-mediated OC differentiation.

Currently, there is no clear explanation for the opposing activities of Vav1 and Vav3 in OC differentiation and bone resorption. We speculate that Vav1 may co-exist with Vav3 and share common target molecules in OCs, and that Vav1 may inhibit or counterbalance Vav3-mediated OC differentiation by competitively inhibiting Vav3 binding to their common target molecules in response to "outside-in" signaling from extracellular signals. Vav1 deficiency may increase the effects of Vav3 on OC differentiation and bone resorption as observed in the femurs of Vav1 $1^{-1-}$ mice. Rho-GEF kalirin has been reported to play a similar role to Vav1 in maintaining bone mass by controlling OC differentiation. Deletion of kalirin results in the decrease in the cortical bone mass of mice (32). Genomic studies have suggested that the function of Vav isoforms may have co-evolved with tyrosine kinase pathways to convert extracellular signals into biological responses in a competitive manner (33).

In summary, this study demonstrates that Vav1 suppresses OC differentiation, as shown by the increased potential of
Vav1 ${ }^{-l-}$ BMMCs to differentiate into OCs than WT BMMCs. This was confirmed by the increased number of mature TRAP-positive OCs in the low density femoral bones of Vav1 ${ }^{-1-}$ mice. Moreover, Vav1 inhibited the adhesion of OC precursors to vitronectin. Therefore, Vav1 may prevent bone resorption by inhibiting the interaction between OCs and bone tissues via integrins in order to maintain bone mass.

\section{MATERIALS AND METHODS}

\section{Reagents}

M-CSF and RANKL were purchased from PeproTech (London, UK). Phosphate-buffered saline (PBS), $\alpha$-minimum essential medium ( $\alpha$-MEM), and fetal bovine serum (FBS) were obtained from HyClone (Logan, UT). PCR primers were procured from Bioneer (Daejeon, Korea). All other chemicals were purchased from Sigma (St, Louis, MO).

\section{Mice}

$\mathrm{Vav} 1^{-/-}$mice were generated previously (34) and the C57BL/6J mice used as the WT control were purchased from Jackson Laboratory (Bar Harbor, ME). Mice were housed under pathogen-free conditions in the animal facility of Inha University. All procedures were conducted in accordance with the institutional guidelines approved by the Animal Care and Use Committee of Inha University (INHA 161214-465). To isolate bone marrow cells, mice were sacrificed by cervical dislocation and every effort was made to minimize their suffering.

\section{Osteoclast differentiation from bone marrow cells}

Bone marrow cells were prepared from 6 to 8 -wk-old male mice and cultured overnight in $\alpha$-MEM supplemented with $10 \%$ FBS and $10 \mathrm{ng} / \mathrm{ml}$ M-CSF. Non-adherent cells were separated and cultured continuously with $30 \mathrm{ng} / \mathrm{ml}$ M-CSF for more 3 days to induce differentiation into OC precursors. These cells were cultured for an additional 4 days in the presence of $30 \mathrm{ng} / \mathrm{ml} \mathrm{M-CSF}$ and $50 \mathrm{ng} / \mathrm{ml}$ RANKL. The formation of multinucleated functional OCs was assessed by counting the number of TRAP-positive cells containing more than three nuclei.

\section{TRAP staining}

We stained TRAP-positive cells using a Leukocyte Acid Phosphatase Assay kit according to the manufacturer's instructions. Briefly, cells were washed with PBS and fixed using a fixative solution containing citrate solution, acetone, and $37 \%$ formaldehyde. Fixed cells were treated with TRAP staining solution for $60 \mathrm{~min}$ at $37^{\circ} \mathrm{C}$ and washed twice with water. After counterstaining with hematoxylin, the number of TRAP-positive cells containing three or more nuclei was counted. 


\section{Analysis of TRAP activity}

TRAP enzyme activity was determined as described previously (35). Cells were incubated with $10 \mathrm{mM}$ sodium tartrate and 5 $\mathrm{mM}$ p-nitrophenyl phosphate in $50 \mathrm{mM}$ citrate buffer $(\mathrm{pH} 4.5)$ for $30 \mathrm{~min}$ at $37^{\circ} \mathrm{C}$. The enzyme reaction was then terminated with $0.1 \mathrm{~N} \mathrm{NaOH}$. Absorbance was measured at $405 \mathrm{~nm}$ using a VersaMax microplate reader (Molecular Devices, Sunnyvale, CA) equipped with SoftMax software.

\section{RT-PCR and quantitative PCR}

Total RNA was extracted from the OCs using TRI reagent (MRC, Cincinnati, OH) according to the manufacturer's instructions, and then reverse transcribed according to the protocol provided by Takara Bio (Japan). The PCR amplification of Vav1 mRNA was performed using the following primers; forward, 5'-GAC GAA GAT ATT TAC AGT GG-3' and reverse, 5'-GCT TAT CAT ACT CTG TCA TC-3'. Quantitative real time (qRT)-PCR was carried out on an Applied Biosystems StepOne unit (Foster City, CA) with SYBR Green PCR Master Mix (Toyobo, Japan) and the following primers (forward and reverse, respectively): TRAP, 5'-ACG GCT ACT TGC GGT TTC A-3' and 5'-TCC TTG GGA GGC TGG TCT T-3'; CTSK, 5'-GAA GAA GAC TCA CCA GAA GCA G-3' and 5'-TCC AGG TTA TGG GCA GAG ATT-3'; CTR, 5'-TGC TGG CTG AGT GCA GAA ACC-3' and 5'-GGC CTT CAC AGC CTT CAG GTA C-3'; DC-STAMP, 5'-TCCTCCATGAACAAACAGTTCCA A-3' and 5'-AGACGTGGTTTAGGAATGCAGCTC-3'; GAPDH, 5'-CCT TCC GTC CTA CCC C- $3^{\prime}$ and 5'-CCC AAG ATG CCC TTC ATG-3' (35). Amplification data were analyzed using the sequence detection software provided by Applied Biosystems.

\begin{abstract}
Adhesion assay
OC precursors were seeded in 48-wells plates coated with 10 $\mu \mathrm{g} / \mathrm{ml}$ vitronectin (R\&D Systems, Minneapolis, MN) for 10 and $60 \mathrm{~min}$ at $37^{\circ} \mathrm{C}$. Unattached cells were rinsed with PBS, stained with Diff-Quick solutions (Thermo, Rockford, IL), observed under the Axioskop2 Plus microscope (Carl Zeiss, Oberkochen, Germany), and then the number of adhered cells was counted.
\end{abstract}

Histology and microcomputed tomography $(\mu \mathrm{CT})$ analysis The femurs of 6-wk-old male mice were fixed with $10 \%$ formalin, decalcified with $10 \%$ EDTA, dehydrated, embedded in paraffin, and sliced into $5 \mu \mathrm{m}$ sections. The sections were either stained for TRAP or hematoxylin and eosin. The trabecular microarchitecture of the distal femoral metaphysis was determined using SkyScan $\mu \mathrm{CT}$ (Brucker, Belgium) at 80 $\mathrm{kV}, 80 \mu \mathrm{A}$, and a $6.5-\mu \mathrm{m}$ voxel size. Cortical bone parameters such as, BV/TV, Tb.Th, Tb.Sp, and Tb.N were analyzed by $\mu \mathrm{CT}$ at $3000 \mathrm{~ms}$ per projection (500 projections in total) (36).

\section{Western blot analysis}

Lysates from OCs were prepared as described previously (37) and electrophoresed on $10 \%$ SDS-PAGE. The resolved proteins were transferred onto a PVDF membrane (Millipore, Bedford, MA) and probed with an antibody to Vav1 (C-14; Santa Cruz Biotech. Dallas, TX). Immunoblots were developed using ECL reagent (Thermo).

\section{Statistical analyses}

Two-tailed Student's t-test (paired) was performed using Microsoft Excel (Redmond, WA). Data are expressed as the mean \pm standard deviation $(\mathrm{SD})$ and $p$ values of $\mathrm{P}<0.05$ were considered significant.

\section{ACKNOWLEDGEMENTS}

We would like to thank Yutie Wang for his technical assistance. This work was supported by the National Research Foundation of Korea (NRF-2014R1A1A2057148 and NRF2017R1A2B4007185).

\section{CONFLICTS OF INTEREST}

The authors have no conflicting interests.

\section{REFERENCES}

1. Katzav S, Martin-Zanca D, Barbacid M (1989) vav, a novel human oncogene derived from a locus ubiquitously expressed in hematopoietic cells. EMBO J 8, 2283-2290

2. Gakidis MA, Cullere X, Olson T et al (2004) Vav GEFs are required for $\beta 2$ integrin-dependent functions of neutrophils. J Cell Biol 166, 273-282

3. Krawczyk C, Oliveira-dos-Santos A, Sasaki T et al (2002) Vav1 controls integrin clustering and MHC/peptidespecific cell adhesion to antigen-presenting cells. Immunity $16,331-343$

4. Turner M and Billadeau DD (2002) VAV proteins as signal integrators for multi-subunit immune-recognition receptors. Nat Rev Immunol 2, 476-486

5. Pearce AC, McCarty OJ, Calaminus SD, Vigorito E, Turner M, Watson SP (2007) Vav family proteins are required for optimal regulation of PLC $\gamma 2$ by integrin $\alpha$ llb $\beta 3$. Biochem J 401, 753-761

6. Garcia-Bernal D, Wright N, Sotillo-Mallo E et al (2005) Vav1 and Rac control chemokine-promoted T lymphocyte adhesion mediated by the integrin $\alpha 4 \beta 1$. Mol Biol Cell 16, 3223-3235

7. del Pozo MA, Schwartz MA, Hu J, Kiosses WB, Altman A, Villalba M (2003) Guanine exchange-dependent and -independent effects of Vav1 on integrin-induced $\mathrm{T}$ cell spreading. J Immunol 170, 41-47

8. Spurrell DR, Luckashenak NA, Minney DC et al (2009) Vav1 regulates the migration and adhesion of dendritic cells. J Immunol 183, 310-318

9. Manolagas SC (2000) Birth and death of bone cells: basic regulatory mechanisms and implications for the pathogenesis and treatment of osteoporosis. Endocr Rev 21, 115-137

10. Koh JM (2018) Osteoclast-derived SLIT3 is a coupling 
factor linking bone resorption to bone formation. BMB Rep 51, 263-264

11. Roodman GD (1996) Advances in bone biology: the osteoclast. Endocr Rev 17, 308-332

12. Athanasou NA (1996) Cellular biology of bone-resorbing cells. J Bone Joint Surg Am 78, 1096-1112

13. Vaananen HK and Laitala-Leinonen T (2008) Osteoclast lineage and function. Arch Biochem Biophys 473, 132-138

14. Holtrop ME and King GJ (1977) The ultrastructure of the osteoclast and its functional implications. Clin Orthop Relat Res 123, 177-196

15. Nesbitt S, Nesbit A, Helfrich M, Horton M (1993) Biochemical characterization of human osteoclast integrins. Osteoclasts express $\alpha v \beta 3, \alpha 2 \beta 1$, and $\alpha v \beta 1$ integrins. J Biol Chem 268, 16737-16745

16. Mellis DJ, Itzstein C, Helfrich MH, Crockett JC (2011) The skeleton: a multi-functional complex organ: the role of key signalling pathways in osteoclast differentiation and in bone resorption. J Endocrinol 211, 131-143

17. Faccio R, Teitelbaum SL, Fujikawa K et al (2005) Vav3 regulates osteoclast function and bone mass. Nat Med 11, 284-290

18. Saftig P, Hunziker E, Wehmeyer O et al (1998) Impaired osteoclastic bone resorption leads to osteopetrosis in cathepsin-K-deficient mice. Proc Natl Acad Sci 95, 13453-13458

19. Hattersley G and Chambers TJ (1989) Calcitonin receptors as markers for osteoclastic differentiation: correlation between generation of bone-resorptive cells and cells that express calcitonin receptors in mouse bone marrow cultures. Endocrinol 125, 1606-1612

20. Perilli E, Baleani M, Ohman C, Fognani R, Baruffaldi F, Viceconti M (2008) Dependence of mechanical compressive strength on local variations in microarchitecture in cancellous bone of proximal human femur. J Biomech 41, 438-446

21. Hotokezaka H, Sakai E, Kanaoka K et al (2002) U0126 and PD98059, specific inhibitors of MEK, accelerate differentiation of RAW264.7 cells into osteoclast-like cells. J Biol Chem 277, 47366-47372

22. Li X, Udagawa N, Itoh K et al (2002) p38 MAPK-mediated signals are required for inducing osteoclast differentiation but not for osteoclast function. Endocrinol 143, 3105-3113

23. Matsumoto $M$, Sudo T, Saito T, Osada $H$, Tsujimoto $M$ (2000) Involvement of p38 mitogen-activated protein kinase signaling pathway in osteoclastogenesis mediated by receptor activator of NF-kappa B ligand (RANKL). J Biol Chem 275, 31155-31161

24. Boyle WJ, Simonet WS, Lacey DL (2003) Osteoclast differentiation and activation. Nature 423, 337-342

25. Walsh MC, Kim N, Kadono $Y$ et al (2006) Osteoimmunology: interplay between the immune system and bone metabolism. Annu Rev Immunol 24, 33-63

26. Tybulewicz VL, Ardouin L, Prisco A, Reynolds LF (2003) Vav1: a key signal transducer downstream of the TCR. Immunol Rev 192, 42-52

27. Movilla N, Dosil M, Zheng Y, Bustelo XR (2001) How Vav proteins discriminate the GTPases Rac1 and RhoA from Cdc42. Oncogene 20, 8057-8065

28. Lee NK, Choi HK, Kim DK, Lee SY (2006) Rac1 GTPase regulates osteoclast differentiation through TRANCE-induced NF- $\kappa$ B activation. Mol Cell Biochem 281, 55-61

29. Movilla N and Bustelo XR (1999) Biological and regulatory properties of $\mathrm{Vav}-3$, a new member of the Vav family of oncoproteins. Mol Cell Biol 19, 7870-7885

30. Wang $Y$, Lebowitz D, Sun $C$, Thang $H$, Grynpas MD, Glogauer M (2008) Identifying the relative contributions of Rac1 and Rac2 to osteoclastogenesis. J Bone Miner Res 23, 260-270

31. Roberts AW, Kim C, Zhen L et al (1999) Deficiency of the hematopoietic cell-specific Rho family GTPase Rac2 is characterized by abnormalities in neutrophil function and host defense. Immunity 10, 183-196

32. Huang S, Eleniste PP, Wayakanon K et al (2013) The Rho-GEF Kalirin regulates bone mass and the function of osteoblasts and osteoclasts. Bone 60, 235-245

33. Bustelo XR (2001) Vav proteins, adaptors and cell signaling. Oncogene 20, 6372-6381

34. Fischer KD, Kong $\mathrm{YY}$, Nishina $\mathrm{H}$ et al (1998) Vav is a regulator of cytoskeletal reorganization mediated by the T-cell receptor. Curr Biol 8, 554-562

35. Kang IS and Kim C (2016) NADPH oxidase gp91phox contributes to RANKL-induced osteoclast differentiation by upregulating NFATc1. Sci Rep 6, 38014

36. Bouxsein ML, Boyd SK, Christiansen BA, Guldberg RE, Jepsen KJ, Muller R (2010) Guidelines for assessment of bone microstructure in rodents using micro-computed tomography. J Bone Miner Res 25, 1468-1486

37. Kim C and Dinauer MC (2001) Rac2 is an essential regulator of neutrophil nicotinamide adenine dinucleotide phosphate oxidase activation in response to specific signaling pathways. J Immunol 166, 1223-1232 